\title{
PREPARATION OF RADIOACTIVE TARGETS FOR CHARGED PARTICLE NUCLEAR SPECTROSCOPY AT THE CERN-ISOLDE PROJECT
}

\author{
R.A. Naumann, D. Burke, H. Ravn
} CERN-ISOLDE

G. Hlawatsch, T. von Egidy

Physics Department, Technical University, Munich

$$
\text { J. de Boer }
$$

Physics Department, Ludwig Maximalians University, Munich and the ISOLDE Collaboration, CERN, $\mathrm{CH}-1211$ Geneva 23

\begin{abstract}
The application of charged particle nuclear transfer spectroscopy to radioactive targets is a powerful technique for establishing detailed properties of nuclei off the stability line. The high intensities of many diverse radioactive beams now available at the ISOLDE-2 separator makes it attractive to initiate such research which, as yet, is unrepresented at CERN.

We report here the results of a survey experiment intended to demonstrate the possibility of preparing suitable targets at CERN and then transporting these to a laboratory equipped with a suitable accelerator and charged particle spectrometer necessary for such measurements.
\end{abstract}

To be presented at:

Eleventh Int. Conf. on Electromagnetic Isotope Separators and Techniques Related to Their Applications, Los Alamos, N.M. 18-22 August, 1986 


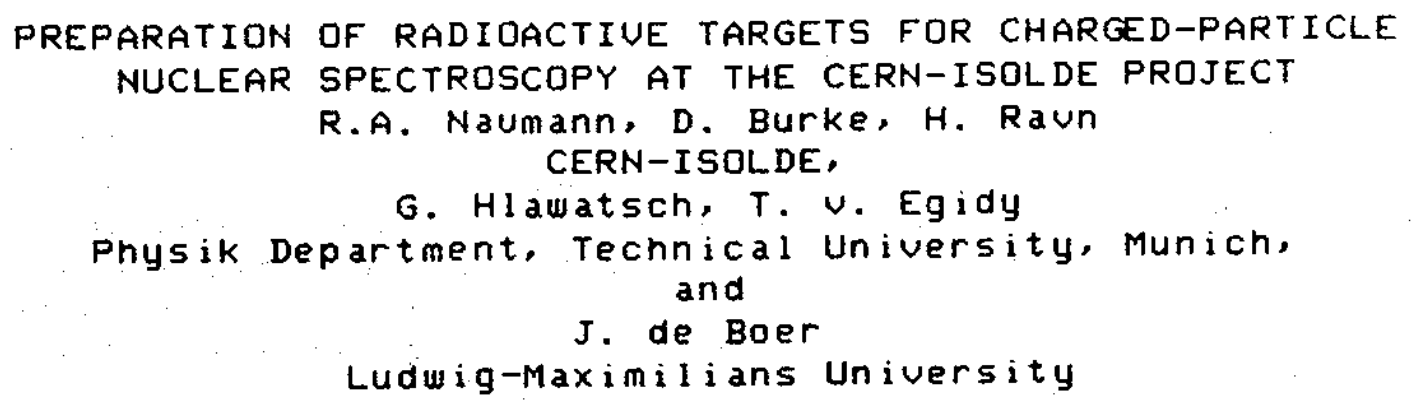

The application of charged-particle nuclear transfer spectroscopy to radioactive targets is a powerful technique for establishing detailed properties of nuclei off the stability line. The high interisities of many of the diverse radioactive beams now available at the ISOLDE-2 separator makes it attractive to initiate such research which as yet is unrepresented at CERN.

We report here the results of a survey experiment intended to demonstrate the possibility of preparing suitable targets at CERN and then transporting these to a laboratory equipped with a suitable accelerator and charged-particle spectrometer necessary for such measurements.

Three main areas should be considered in connection with such an effort.

(a) the production of the necessary quantities. of radio isotopes,

(b) the deposition of the radioactive isotopes on the required thin carbon foils at the ISOLDE separator, and

(c) the transportation and handing of the quite radioactive and fragile foils both at CERN and at the site of the charged-particle spectroscopy.

We take up these areas sequentially, and finally report on the data obtained with an ISOLDE-produced radioactive target at the Garching-Munchen Uan de Graaff Accelerator Laboratory.

Requirements and Production of the Radioisotopes

A $t$ arget of some nerium mass (An1OO) isotope suitable for transfer spectroscopy has a thickness of $25 \mu \mathrm{g} / \mathrm{cm}$ extending over a cross-sectional area of $2 \times 3 \mathrm{~mm} 2$. One tenth of this thickness is at the limits of practicality. These characteristics respectively correspond to total quantities of 1.50 to 0.15 micrograms; in the present case, $9 \times 1015$ or $9 \times 1014$ radioactive atoms. Appendix I prouides the yields now auailable at ISOLDE-2 for a nigh-yield element (Cesium); a production rate of 1012 atoms per second with a $3 \mu A$ beam from the synchrocyclotron is typical. Accordingly, one may expect to deposit the required material in collection periods of the order of 1 day (B.6) 604 seconds).

The activity and radiation levels involved when working with such targets require consideration. Assuming the production rate $R=1011$ atoms/second, one estimates the activity level of the collected target in terms of the ratio of the collection $t i m e$ t 
to the half-life Th as:

$$
A=\ln 2 R(t / T H)
$$

i.e. for a one-day collection of radioisotopes with respective half-lives of 10 days, 100 days, 1000 days activity levels of 190 mc, $19 \mathrm{mc}$, $1.9 \mathrm{mc}$ levels are involved. For a hard gamma-emitting isotope, this corresponds very approximately to radiation levels of 200, $20,2 \mathrm{mR} / \mathrm{hr}$ at orie meter -- levels that can be handled for short periods of tinie using lead shielding walls installed in a standard radiochemical hood. One should also note that such quantities of radioisotopes are already commonly transported for medical use.

The choice of the four cesium isotopes involved in our trial experiments was primarily based on their high yields and convenient radiation properties:

$$
\begin{array}{ll}
131 \mathrm{Cs}(T K=9.7 d) & 132 \mathrm{CS}(T K=6.5 d) \\
134 \mathrm{CS}(T K=2.06 y) & 135 \mathrm{Cs}(T K=2.3 \times 106 \mathrm{y})
\end{array}
$$

Cesium 131 is attractive for two reasons: (i) $98 \%$ of its electron capture events populate the ground state of Xenon 131 . Thus, the main radiation associated with this radioisotope are easily shielded $30 \mathrm{keU}$ Xe $k \times-r$ ays; (ii) should a spill occur, within two months residual radiation levels would diminish by 2 orders of magnitude. The decay of cesium 132 populates a 667 keU level of Xenon 132 in $96 \%$ of the electron capture events. The deexcitation gamma rays lie very close in energy to the 661 keU photons emitted in Cesium 137 decay. This isotope was collected and retained at CERN for assay purposes. It was not snipped because of the weight of the required heavier shielding. Cesium 134 and 135 were good candidates for collection and shipping because of their collsiderably longer half-life. The Cesium 135 target was not shipped by air; its very long half-life permitted this sample to be treated as a stable isotope and handled or shipped at leisure without restrictions.

The thin carbon foils supporting the radioisotopes used in our trial have a thickness and area typical for charged-particle spectroscopy - 30 lig/cme. This was supported on steel target holders $25 \mathrm{~mm} \times 13 \mathrm{~mm} x 0.1 \mathrm{~mm}$ with an oval aperture $12 \mathrm{~mm} \times 9$ mm. Such thin and tiansparent carbon foils are veryeasily ruptured during handling and transportation leading to foil loss and contamination prolliems. These foils were prepared by Frau Nacke and Dr. Maier-Komor at the thin target laboratory of the Technical University, Munich.

For shipment of the thin foils, a double enclosure based on glass sample vials was developed. The target frame with carbon foil was first supported in a slotted rubber stopper. This was inserted into the smaller vial of dimensions $40 \mathrm{~mm} x 20 \mathrm{~mm}$ containing a conducting graphitized sponge at its bottom. This enabled the target frame to be supported both at its top and bottom. The first sealed assembly was then placed in the second vial with dimensions of $50 \mathrm{~mm} \times 29 \mathrm{~mm}$ also containing a graphitized sponge cushion to provide shock mounting. The second seal was made with a normal plastic top so that the first uial was firmly supported between the top and cushion. The interior of the first vial was copper coated with a semitransparent film and the rubber stopper equipped with a thin aluminum foil tab to 
contact the frame and ilterior of the first vial. This prouided a conduction path preventing possible charge accumulation and destruction of the radioactive, targets.

Figures 1 and 2 respectively show an early and final assembly used for transporting the targets. For the air shipments, each glass vial assembly supporting one foil target was contained in a standard CERN ISOLDE radioisotope shipping container. These consist of a standard central lead pot uith $3 \mathrm{~cm}$ thick walls which is supported in a $38.5 \mathrm{~cm} \times 38.5 \mathrm{~cm} \times 46 \mathrm{~cm}$ wooden shipping container. The glass vial assembly was placed in a paper tissue bed inside the lead pot to provide additional shock cushioning.

One air shipment from CERN to Garching was made with inert foils to prove the air transportation. Subsequently, two active foils (131Cs and $134 \mathrm{Cs}$ ) were air shipped. One glas vial assembly containing an inert foil was even shipped using normal mail from Garching to CERN. All five individual foil trips were successful; no foil breakage occured in any of these trials.

It is important that the active air shipments proceed directly from Geneva to lunich. This avoids the usual off-loading involved in the usual German customs and entry procedures for air-shipped radioactive materials which normally takes place at Frankfurt airport. To accomplish this, special permission uas required from the Luftfahrt-Bundesamt in Brauschweig. Dr. Herring, heading the Physics Department Administration at the Technical University of Munich at Garching, and Herr Boxhorn of the Physics Radiation Protection Department there contributed greatly by securing this permission. The cooperation and help of $\mathrm{Mr}$. Duding and Mi. Scharen at the Lufthansa Airfreight Department, Cointrin Airport Geneva was essential in shipping the radioactive samples on a direct Lufthansa flight from Geneva to Munich.

Deposition of the Radioisotope Samples

The deposition was made using an existing target collection assembly which mounts on a carriage-rail system in the suitchyard region of ISOLDE-2. The position of the carriage is remotely controlled from the ISOLDE control console. For the active targets, the collection assembly was initially positioned behind a metal-shadowing shield to avoid sputtering and unwanted deposition during initial separator tune-up and focussing operations.

The individual target frames bearing the graphite foils were individually mounted on four target blocks which fitted into the target assembly. The relative position of each block was adjusted to the expected relative mass spacings expected from the ISOLDE tabulation. The botton of each target block was insulated with a lucite block and equipped with a current lead. Using an electrometer, the ioll current striking each target could be monitored at the ISOLDE console. Two tests were made of the collection parameters using stable beams.

To check the mass spacing, beams of stable xenon isotopes were used. Millimeter graph paper was mounted on the front of the collection assembly and a "burn-mark" pattern recorded with the separator set for a central mass of 128 . The calculated front positions expected for the mass 129, 131,132 and 134 xenon isotopes were $13.78,39.33,51.78$ and 76.19 mms corresponding to the separations $25.55,12.45$ and $24.41 \mathrm{mms}$. Experimental spacings 
of 25,13 and $24 \mathrm{mms}$ illoicated that the tabulations were reliable within experimental erlor. A second experiment involved a collection of stable cesium 133 on an actual mounted carbon foil to check the collection efficiency. In all of our experiments, the collections have been made using full-energy Go kilovolt ion beams without electrostatic retardation. This made it possible to collect the four cesium isotopes simultaneously using the existing collector assembly. However, sputtering losses must be considered.

To check these, we collected over a period of 1 hour with an ion beam that should have deposited a typical quantity -- 1 microgram of Cesium 133. The collected cesium was assayed using activation analysis kinily arranged by Dr. Wyttenbach at the Swiss Reactor Institute at Wurenlingen. This analysis revealed that $1.2( \pm 10 \%)$ micrograins had indeed been deposited; however, an additional $50( \pm 30 \%)$ miclograms of copper was discovered. Since a copper collimator with a circular aperture $5 \mathrm{~mm}$ in diameter had been used to restrict the collected ion beam, this result indicated that precautions had to be taken to minimize sputtering. "Hence, we used graphite-edged beam collimators and enclosed each target foil as thoroughly as possible. We also introduced the shadowing shield in the switchyard tank to hide the collection assembly during initial separator tune-up.

Figure 3 shows the target block assembly used for the collection. The graphite-lined collimator prouiding a $5 \mathrm{~mm}$ diameter aperture is clearly shown. Benind the collimator the target frame, slotted for mounting screws, just protrudes. To the right of the target block a thin aluminum shield is shown. This closely surrounded the foils during collection and was shadowed from the impinging beams by the front collimator. A back shield positioned behind the target frame is not visible in this photograph.

For the radioactive collections, a central mass of 130 was chosen. The position of the center of the mass 131 beam (front mounted) lies 12.83 min from this point. Similariy, the mass 132 (back mounted) and 134 (fiack mounted), respectively, lay at 28.98 $\mathrm{mm}$ and $56.39 \mathrm{~mm}$, while the front mounted mass 135 beam lay at $63.18 \mathrm{~mm}$. All collection blocks were positioned on the collection assembly so that the center of mass 131 foil lay $10.00 \mathrm{~mm} f \mathrm{fom}$ the edge of the collection assembly. (That is, the edge of the coliection assembly uas remotely positioned $2.83 \mathrm{~mm}$ from the central mass position.)

The radioactive collection was made over a 24-nour period. Unfortunately, the ioll currents of cesium isotopes measured from the molten lanthanum target were about one $s i x t h$ of those expected. Most probably, the molten lanthanum target had developed a leak so that the effective target thickness was less than usual. This was confirmed in an assay of the cesium 132 kindly made by Dr. Raffensoe of the CERN Radiation Standards Laboratory. Instead of the expected $340 \mathrm{millicuries,} \mathrm{only} 35$ millicuries of Cesium 132 were collected on a thick aluminum catcher.

\section{Handling. Transportation and Use of Tarqets}

After completion of the collection, the ISOLDE switchyard section was vented to ail. Using step-in overalls, shoe covers, gauze breathing mask and rubber gloves, the switchyard room was entered and the top of the suitchyard tank removed. The target 
assembly containing the carbon foils was quickly detached by hand from the carriage and $p l$ aced in a glass dessicator for transport. The switchyard tank top was replaced, and the dessicator rolled in a lead-shielded target cart to the not laboratory area. Here, a lead-brick enclosure involving a shielding floor and wall had been erected in a hood and covered with paper to minimize lead-dust contaminatioll. This structure includedalead-glass viewing port. Using this enclosure, the dessicator was opened. and the target assemhly dismantled using forceps and a screw driver. The carbon foils were removed from the target blocks and installed in the trarisportation vial assemblies. The Cesium 131 and 134 targets were placed in ISOLDE shipping boxes and after customs clearance at the Munich-Riem airport, were transferred by truck to a hood in the Garching hot laboratory of the LMU Physics Departiment.

The sealed target housing constructed for radioactive target experiments at the QDDD spectrograph is shown in Figure 5 . This involves a central target ladder contained in a sealed enclosure equipped with a lamp and vieuing port. The sealed enclosure can be pumped separately through a line and small filtered uacuum pump. By means of an electric motor rotating tuo worm drives, a bottom cup sealing the enclosure can be lowered to expose the target 1 adder.

With the kind help of Dr. Maier and his assistant at the target laboratory of the LMU Physics Department, the target ladder was loaded. Together with the cesium 131 target, a reference thin gold target, a plain carbon foil target and a focussing aperture were included. The target assembly was then moved to the QDDD spectrograph, installed in the target chamber and pumped out.

To assess the thickness of the cesium 131 target, elastic scattering of $15 \mathrm{MeU}$ dellterons was used. Figure 6 shows the deuteron spectrum recorded at 90 with the gold foil. The peaks corresponding to the gold 197 ground state and three excited states provide an energy calibration. Figure 7 shows the deuteron elastic spectrum obtained with the cesium 131 target and a 1.5 microampere beam. To our knowledge, this spectrum records a result from the shortest-lived radioactive target yet used in a scattering spectroscopic experiment.

The data recorded can be used to confirm the mass of the cesium isotope corresponding to the elastic peak of Figure 8 . At 900 in the laboratory flame when an incident beam of particles with mass $m$ and incident energy $E_{0}$ are elastically scattered from a target of mass 1 , the final energy. Eqo, is given by:

$$
E_{40}=\frac{(M-m)}{M+m} E_{0}
$$

For mass 131, one expects the deuteron elastic peak will nave an energy 149 keU lower than the gold elastic peak. This corresponds to channel 290 in agreement with observation.

unfortunately, before the less intense deuteron peaks could be recorded, a tear developed in the cesium 131 graphite foil after about 1 hour of irradiation. It is not yet clear whether this tearing was due to chemical instability of cesium deposited on graphite, too high a beam current or was associated with a large water leak that had occurred in the ODDD target chamber 
immediately before our experiment.

The target assembly was then sealed and removed from the QDDD target chamber using bagging techniques in case of possible activity spread. A detailed wiping test subsequently revealed absolutely no loose actiuity in the QDDD target chamber.

We finally wish to record here our gratitude for the appreciated and friendly cooperation of the radiation protection services at CERN and the Garching Accelerator Laboratory. Without the essential help of li. Del Tenre at CERN and Herr Birnstock at Garching together with their staffs in all aspects of target handing, this trial expleriment would not have been possible.

\section{To Summarize:}

The main problems associated with the preparation of radioactive targets at ISOLDE for transfer spectroscopy and transporting them to an appropriate laboratory have been explored. We believe that the practicality of such an effort has been demonstrated. 


\begin{abstract}
Appendix I
CS ISOTOPE PRODUCTION (after H. RaUn)

[ $140 \mathrm{~g} / \mathrm{cm}^{2}$ La Target; $600 \mathrm{MeU}$ p $3 \mu \mathrm{\mu}$, Ta Surface Ionization]
\end{abstract}

MASS

129

130

131

132

133

134

135

136

137
HALF LIFE

$$
\begin{gathered}
32.1 \mathrm{~h} \\
29.2 \mathrm{~m} \\
9.7 d \\
6.5 d \\
5 t a b l e \\
2.1 y \\
2 \times 106 y \\
13.2 d \\
30.2 y
\end{gathered}
$$

YIELD (dps)
2. $55 \times 10^{11}$
1. $77 \times 10^{11}$
1. $50 \times 10^{11}$
1. $17 \times 10^{11}$
8. $40 \times 1011$
5. $70 \times 1010$
3. $90 \times 1010$
2. $13 \times 1010$
$6 \times 10^{9}$

\title{
Appendix II
}

At the ISOLDE facility an electrostatic retardation and collection system is being planned by Jon Petersen. Such a facility applied to the preparation of radioactive targets offers the attractive possibilities of preparing thicker targets through better focussing and avoiding sputtering losses. A gated and separately removable retardation and collection unit would significantly aid the transportation of the active targets collected and minimize possible contamination hazards in the UR area. 


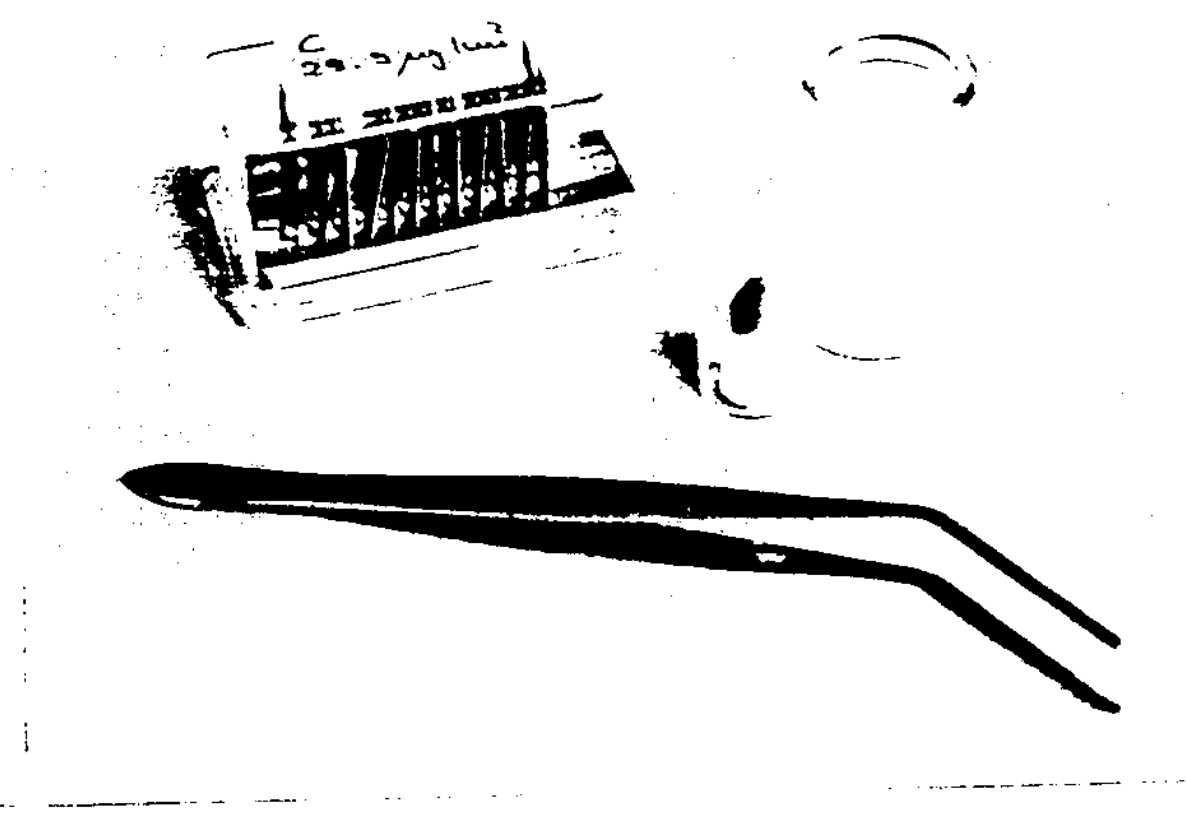

Figure 1. Early Shipping Vial Assembly

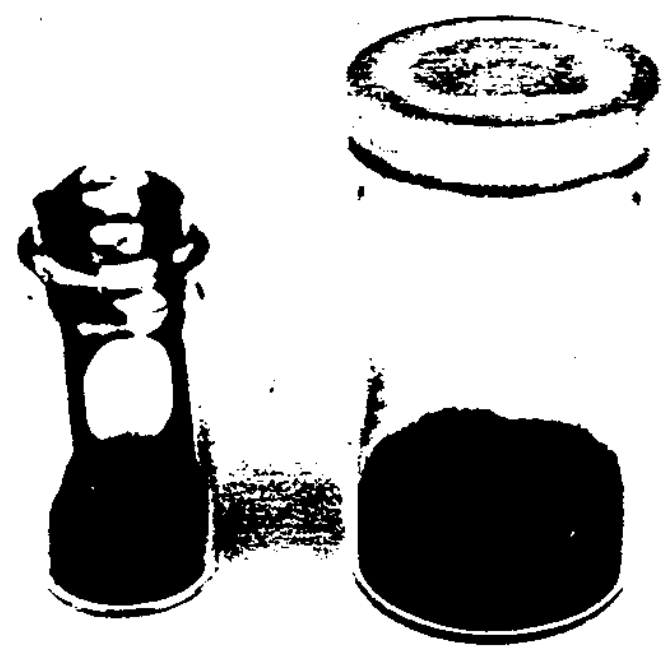

Figure 2. Late Snipping Uial Assembly 

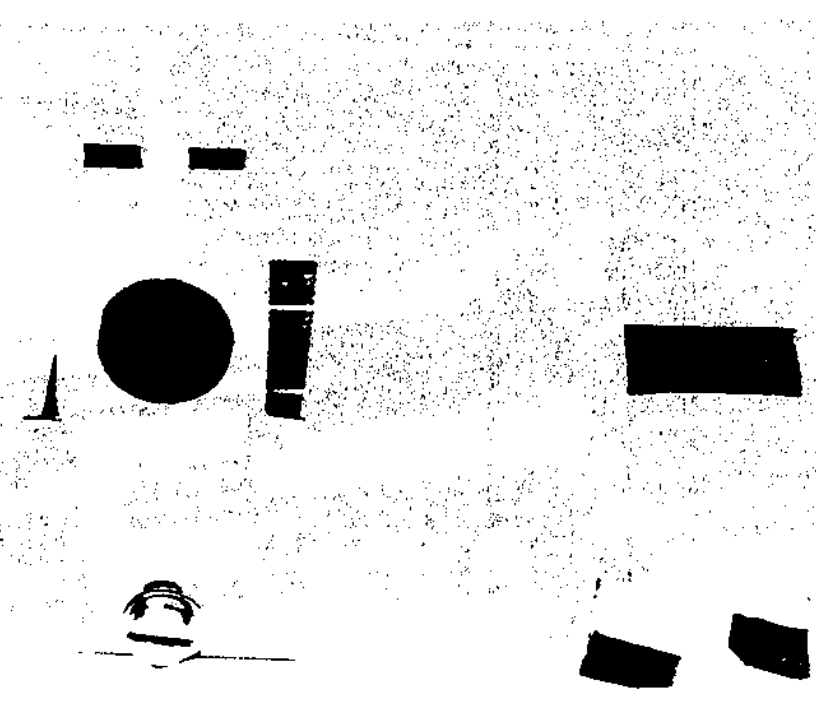
Figure 3. Target Block Assembly Used for
Radioactive Collections

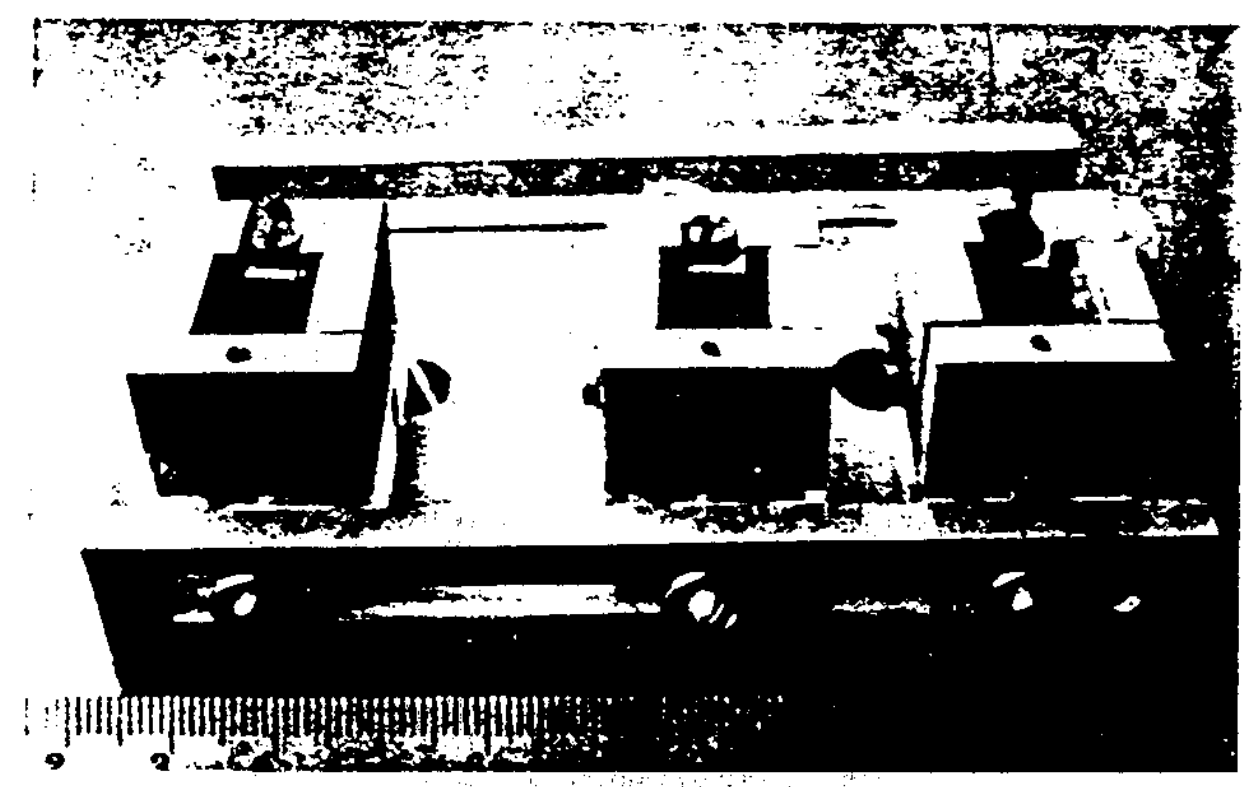

Figure 4. ISOLDE Collection Assembly Carriage 


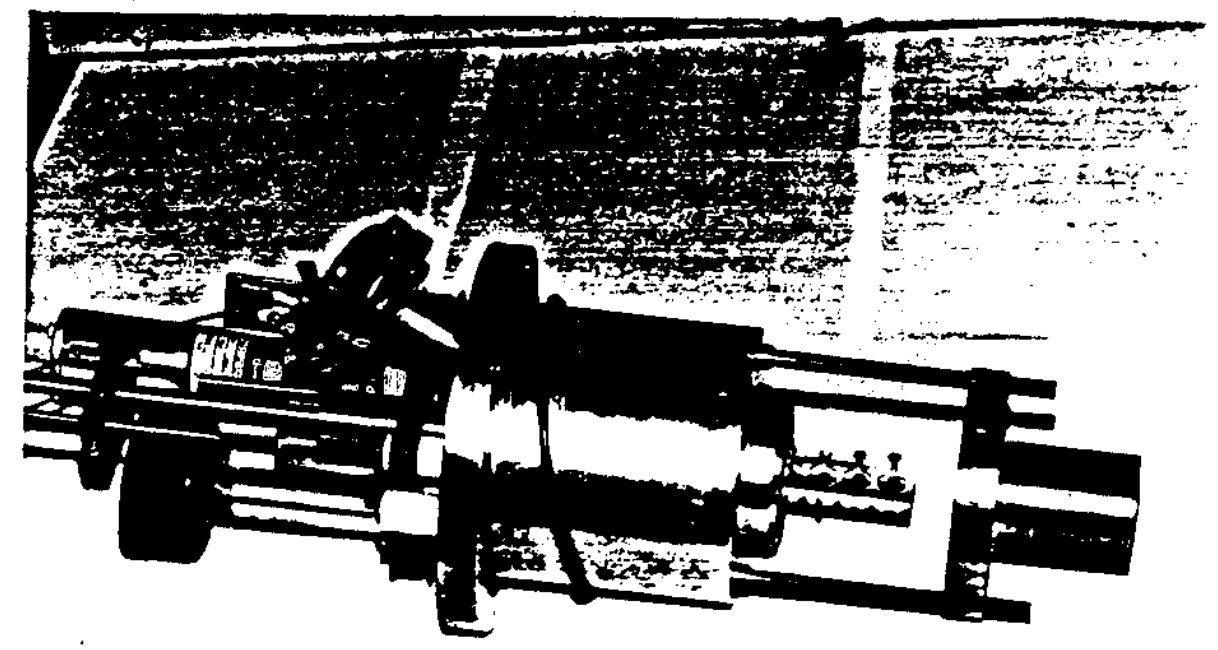

Figure 5. Target Housing for ODDD Experiments

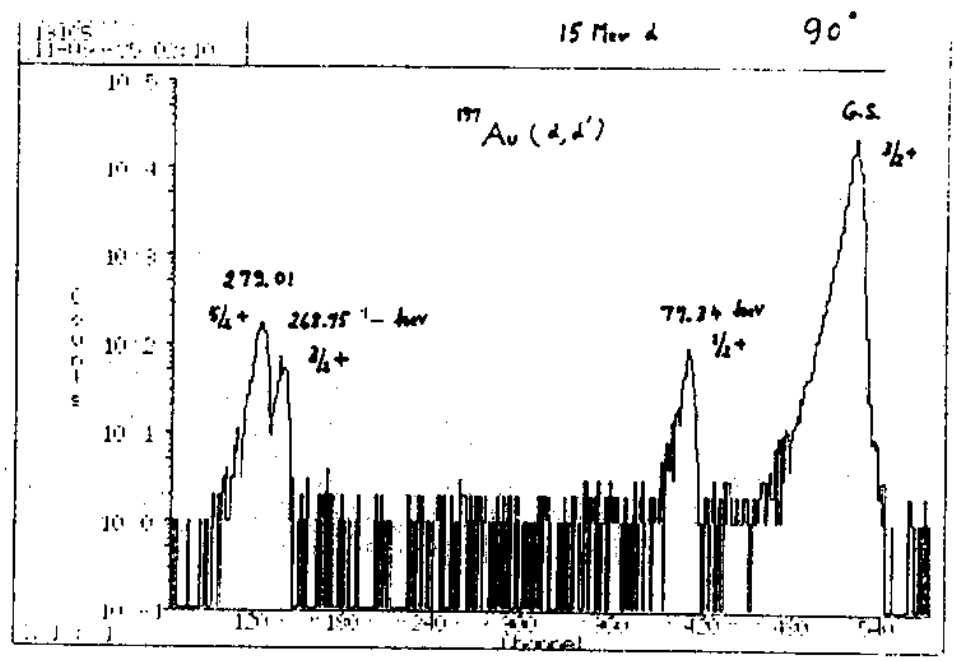

Figure 6. Inelastic Deuteron Scattering from Gold 197, 90, $15 \mathrm{MeU}$ 


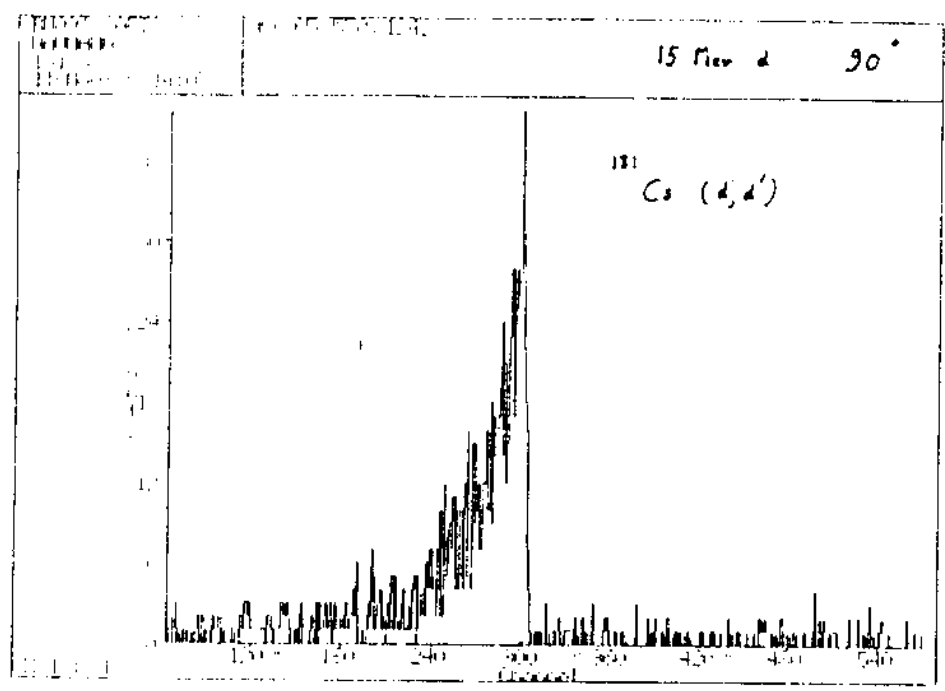

Figure 7. Inelastic Deuteron Scattering from Cesium 131, 90, $15 \mathrm{MeU}$ 